\title{
Lattice Boltzmann in micro- and nano-flow simulations
}

\author{
Xiaowen SHAN $^{1, *}$ \\ * Corresponding author: Tel.: +1 (781)676-8530; Fax: +1 (781)676-8599; Email: xiaowen@exa.com \\ 1: Exa Corp., 55 Network Drive, Burlington, Massachusetts 01803, USA
}

\begin{abstract}
One of the fundamental difficulties in micro- and nano-flow simulations is that the validity's of the continuum assumption and the hydro-dynamic equations start to become questionable in this flow regime. The lower-level kinetic/molecular alternatives are often either prohibitively expensive for practical purposes or poorly justified from a fundamental perspective. The lattice Boltzmann (LB) method, which originated from a simplistic Boolean kinetic model, is recently shown to converge asymptotically to the continuum Boltzmann-BGK equation and therefore offers a theoretically sound and computationally effective approach for micro- and nano-flow simulations. In addition, its kinetic nature allows certain microscopic physics to be modeled at the macroscopic level, leading to a highly efficient model for multiphase flows with phase transitions. With the inherent computational advantages of a lattice model, e.g., the algorithm simplicity and parallelizability, the ease of handling complex geometry and so on, the LB method has found many applications in various areas of Computational Fluid Dynamics (CFD) and matured to the extend of commercial applications. In this talk, I shall give an introduction to the LB method with the emphasis given to the theoretical justifications for its applications in micro- and nano-flow simulations. Some recent examples will also be reported.
\end{abstract}

Keywords: Micro Flow, Kinetic theory, Lattice Boltzmann

\section{Introduction}

Due to the growing number of scientific and engineering applications, simulation of fluid flows in geometries of micro- and nanometer scales has become an important research area in recent years. Flows in such regime are often characterized by a finite Knudsen number, defined as $K n=l / L$, where $l$ is the mean free path of fluid molecules and $L$ the characteristic length of the geometry. As the Knudsen number also measures the departure from the local equilibrium, at finite Knudsen numbers, the basic assumption of hydrodynamics of near equilibrium ceases to be valid as the characteristic length of the geometry becomes smaller and smaller. The fluid behavior in such confined geometries can not be described by the Navier-Stokes (NS) equations and certain lower-level descriptions and methods, e.g., molecular dynamics, DSMC, or kinetic theory based methods have to be used in these situations. As solving the Boltzmann equation either by direct simulation or by the so-called Direct Simulation Monte Carlo (DSMC) method is still too cost prohibitive for engineering purposes on today's computer, simulations through certain reduced forms of Boltzmann equation, e.g., the discrete-velocity models and the moment equations become very attractive alternatives.

In recent years, the lattice Boltzmann method has received a great amount of attention as a promising method of CFD with a number of higher desirable features Chen and Doolen (1998); Succi (2001, see). Although originates from the Lattice Gas Cellular Automaton (LGA) as a way for mimicking NS fluids, the LB equations has recently been shown to asymptotically approach to the BoltzmannBGK equation. Promising numerical evidences have also emerged in the literature which 
demonstrated LB's capabilities in simulation of micro and nanal flows.

In this presentation, we first give a brief review of the theory of the LB method, with the emphasis on the recent derivation of LB from the continuum kinetic theory, which enables the extension of the LB method to the flow regime of finite Knudsen numbers. The current status of LB application in micro- and nanoflow simulations is then reviewed.

\section{The lattice Boltzmann theory}

\subsection{The development history}

Historically, the LB method is a direct derivative of the Lattice Gas Cellular Automaton (LGA), which was first proposed more then two decades ago as a simple and efficient fluid model using Boolean particles by Frisch et al. (1986) and Wolfram (1986). In this model, fluid motion is simulated as the averaged behavior of fictitious fluid particles on a regular lattice. Depicted in Fig. 1 are the wellknown FHP LGA model of Frisch, Hasslacher, and Pomeau (1986), named after the three inventors. Here, each particle assumes one of the seven velocities represented by the zero vector and the six vectors pointing from the site where the particle resides to one of its six neighbors. The microscopic dynamics consisted of a "streaming" step in which particles hop to one of the neighboring sites according to their velocities, and a "collision" step in which particles at each lattice site are re-distributed according to a set of "collision rules" which satisfies the conservations of mass and momentum. On the surface of a solid wall, the conventional no-slip boundary condition can be approximately achieved in the so-called "bounce-back" scehme by simply reversing the velocities of the incoming particles to send them back to the bulk of the fluid. Like in continuum kinetic theory, the hydrodynamics of such a binary system is obtained by first writing down the kinetic equation with which the Chapman-Enskog calculation was then carried out. The kinetic equation can be written as:

$$
f_{a}\left(\boldsymbol{x}+\boldsymbol{e}_{a}, t+1\right)-f_{a}(\boldsymbol{x}, t)=\Omega_{a}(f)
$$

where $f_{a}$ is the probability of finding one particle at $\boldsymbol{x}$ with velocity $\boldsymbol{e}_{a}$, and $\Omega_{a}(f)$ the collision operator. Eq. (1) has thereafter been known as the lattice Boltzmann equation. The Chapman-Enskog analysis yields a hydrodynamic equations that is similar to the NavierStokes (NS) equation:

$$
\begin{aligned}
& \frac{\partial \rho \boldsymbol{u}}{\partial t}+\nabla \cdot[\rho g(\rho) \boldsymbol{u u}]=-\nabla p \\
& \quad+\nabla \cdot[\nu \nabla(\rho \boldsymbol{u})]+\nabla[\zeta \nabla \cdot(\rho \boldsymbol{u})]
\end{aligned}
$$

where the pressure $p=c_{s}^{2} \rho\left[1-g(\rho) u^{2}\right]$. The most significant differences with the NS equation are the $g(\rho)$ factor in the advection term and the velocity-dependent pressure.

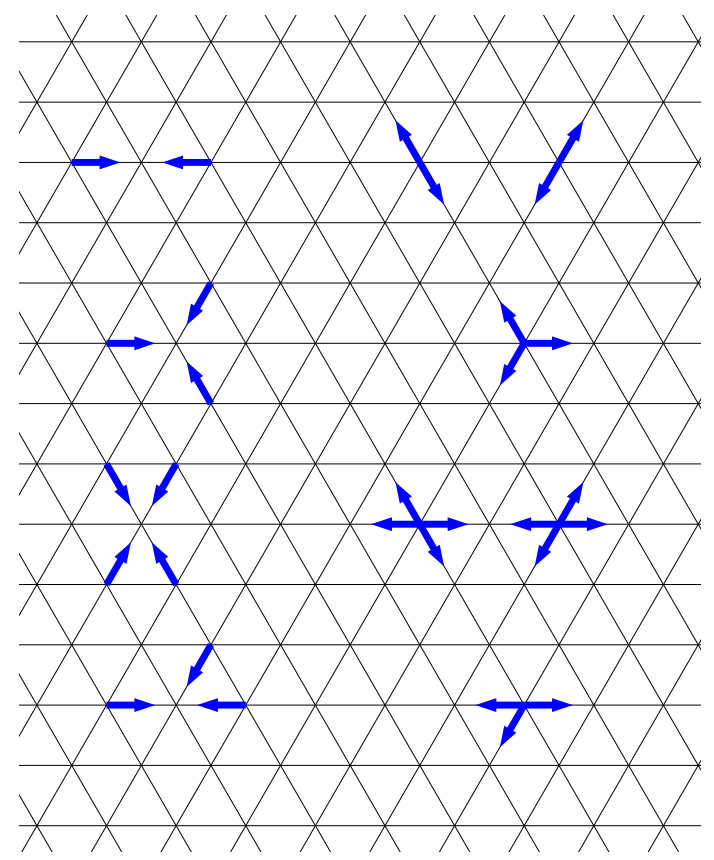

Fig 1: Collision operator in the FHP Lattice Gas Cellular Automaton. For each incoming state on the left column, the possible outcomes are given in the right column.

The deviations from the NS equation, together with the usual statistical noise (Orszag and Yakhot, 1986) associated with all particlelike methods prevented the original LGA from being a useful tool of Computational Fluid 
Dynamics. Nevertheless, it was soon pointed out by McNamara and Zanetti (1988) that the noise problem can be solved by following the lattice Boltzmann equation (1) in computation instead of the Boolean dynamics of the LGA. In practice, this involves the same algorithm of the LGA except that the Boolean particle states are replaces by a real-numbered single-particle distribution function. In addition, the form of the collision operator in Eq. (1) was modified by a number of authors (Higuera and Jimenez, 1989) in attempts to eliminate the deviations from the NS equation. Particularly, as pointed out independently by Chen et al. (1992) and Qian et al. (1992), the macroscopic behavior of the LB equation can be made almost exactly that of the NS equation by adopting the singlerelaxation-time collision model:

$$
\Omega_{a}(f)=-\frac{1}{\tau}\left[f_{a}-f_{a}^{(e q)}\right],
$$

where $f$ is the equilibrium distribution function of the following form:

$$
f_{a}^{(e q)}=w_{a} \rho\left[1+\frac{\boldsymbol{u} \cdot \boldsymbol{e}_{a}}{c_{s}^{2}}+\frac{\left(\boldsymbol{u} \cdot \boldsymbol{e}_{a}\right)^{2}}{2 c_{s}^{4}}-\frac{u^{2}}{2 c_{s}^{2}}\right] .
$$

Eq. (3) was first proposed by Bhatnagar, Gross, and Krook (1954) in their study of continuum kinetic theory and has since been known as the BGK collision term.

In continuum, the equilibrium distribution is the celebrated Maxwell-Boltzmann distribution. In the lattice version, the equilibrium distribution (4) was obtained by first assuming a Taylor expansion in velocity and then determining the expansion coefficients to ensure that the correct NS equation emerge from the Chapman-Enskog calculation. Here in Eq. (4), the form by Qian et al. (1992) for square lattice is given. The equilibrium distribution given by Chen et al. (1992) for a hexagonal lattice differs slightly and we will show that they are essentially identical below. Both the square and the hexagonal models yield the correct NavierStokes equation at the small velocity limit.

Two observations can be made. First, as can be seen from Eq. (4), the velocities are measured by a characteristic velocity which is related to the speed of sound, the small velocity limit physically corresponds to the small Mach number or the near-incompressible limit. Second, by Taylor-expanding the lefthand-side, Eq. (1) can be viewed as a finite difference scheme of the following discretevelocity kinetic equation:

$$
\frac{\partial f_{a}}{\partial t}+\boldsymbol{e}_{a} \cdot \nabla f=-\frac{1}{\tau}\left[f_{a}-f_{a}^{(e q)}\right] .
$$

This observation played important role in the accuracy and stability analysis.

Eqs. (1) and (3)-(4), together with the underlying lattice and a proper set of boundary conditions, defines the basic algorithm of the LB. Significant amount of work has been carried out afterwords on the validation of the LB model, as well as on exploring possible extensions of the LB into other related fields. Most of that work has been summarized by Chen and Doolen (1998) and Succi (2001). By the end of last century, it was well established that the LB system recovers the athermal Navier-Stokes equations in the nearincompressible limit and offers a simple and efficient model for describing isothermal fluid flows in the low-Mach number regime.

Comparing with the conventional hydrodynamic descriptions, e.g., the NS equation, LB method has a number of rather desirable features. First, as evident from Eq. (1), the advection term is a linear operator. Effectively a large time step can be used in time-integration. This allows very efficient solution of timedependent flows. Second, the algorithm is simple, easy to implement, and intrinsically parallelizable. Since the very early days, the LGA was implemented in hardware for maximum performance (Doolen, 1989), and the LB is among the first CFD algorithms that have been implemented on the recent Graphics Processing Unit (GPU) (Li et al., 2003). Third, the implementation of the boundary condition is generally based on the intuitive picture of particle streams. Combined with the Cartesian lattice, the ease in handling complex geometry has made LB almost the only option in simulations of flow through porous media and a 
strong competitor in CFD applications involving complex geometries. Fourth, its kinetic nature allows $\mathrm{LB}$ to be extended to include some of the lower-level physics more easily. An example is that multiphase models were developed since the early days of the LGA and has attracted tremendous amount of attention from various field of science and engineering. In those models, the multiphase phenomenon is simulated by modeling the effect of the intermolecular interaction rather than the motion of the fluid interfaces. This meso-scopic approach has been particularly successful in simulating flow in micro-channels.

Despite the phenomenal success of the LB in a broad area of scientific and engineering applications, some fundamental issues remained.

1. It was discovered at the beginning that although most of the non-physical artifacts were removed in the lattice BGK model, there is still a "cubic" error term that cause the viscosity to depend on the velocity, therefore making the viscous behavior of LB non-Galilean invariant for finite Mach number (Qian and Orszag, 1993; Chen et al., 1994; Qian and Zhou, 1998; Házi and Kávrán, 2006; Nie et al., 2008)

2. The distribution function in the lattice BGK model was obtained by explicitly matching the coefficients of a loworder small-velocity expansion so that the Chapman-Enskog calculation yields the correct NS equation. This procedure presumes small velocity in the starting point and is generally a tedious procedure for the athermal NS equations already. When the energy equation is included in the system, the "matching process" does not always yield a unique solution Alexander et al. (1993) and the thermal LB model so obtained suffers from numerical instability. It was observed by McNamara and Alder (1993); McNamara et al. $(1995,1997)$ that inclusion of more velocities usually improves the numerical stability but generally there is a lack of guideline as to which velocities should be included.

3. It was not at all clear how the LB could be extended to the finite Knudsen number regime as the hydrodynamic equations derived for that regime, e.g., the Burnett and Super Burnett equations are arguably ill-post themselves. Obtaining a LB model with Burnett or Super Burnett behavior is both practically impossible and theoretically unjustifiable.

For these reasons, it was sometimes erroneously asserted that the LB can not be applied to flow regimes other than isothermal nearincompressible flows.

\subsection{The moment theory of $\mathrm{LB}$}

By noticing that the LB equation can be viewed as a finite-difference discretization of the discrete-velocity Boltzmann BGK equation, Abe (1997) and He and Luo (1997) pointed out that the LB equation can be derived from the continuum Boltzmann BGK equation using discrete velocities and a small-velocity Taylor expansion of the Maxwell-Boltzmann distribution as the equilibrium. The discrete velocities must form quadratures in the velocity space so that moments of the Taylor-expanded Maxwellian up to third order can be evaluated using the distribution function values of at those discrete velocities. This condition ensures that the correct isothermal NS equation emerges from the Chapman-Enskog calculation owing to the fact that only the leading moments of the distribution, rather than the entire distribution, are formally used in that calculation. This new derivation of LB is a priori in the sense that it starts from well-established results of kinetic theory and arrives at the LB equations without a posterior determination of the distribution from Chapman-Enskog calculation. Although neither work directly solved any of the aforementioned issues remained in LB theory, this discovery paved the way for a new theoretic frame work within which, those remaining issues have been solved to a satisfactory extent. 
The new LB theory makes a formal link between the LB equations and the Grad 13 moment system. In his seminal paper, in an attempt to find an alternative hydrodynamic theory for flow regimes beyond the NS equations, Grad (1949b) argued that the motion of fluid can be described by the few leading moments of the single-particle distribution function as opposed to the entire distribution itself. In addition to the familiar set of thermo-hydrodynamic variables of density, velocity and temperature, which are themselves the leading moments of the distribution, a few higher moments, i.e., the components of the stress tensor and the heat flux should also be treat in par with the basic set as the dynamic variables. A closure should be sought for the combined thirteen moments. This expansion of dynamic variables can presumably expand the validity domain of the hydrodynamic equations as the Knudsen number is increased. Furthermore, extensions to Grad's moment theory were made to include more moments in simulation of finite Knudsen number flows (Struchtrup, 2002).

Inspired by the Grad 13 moment theory, Shan and He (1998) re-formulated the LB system as an equivalent system to the moment equations. Here we give a sketch of the main derivation. Readers are referred to Shan and He (1998); Shan et al. (2006) for the full details.

Starting from the Boltzmann-BGK equation in continuum:

$$
\frac{\partial f}{\partial t}+\boldsymbol{\xi} \cdot \nabla f=-\frac{1}{\tau}\left[f-f^{(0)}\right]
$$

where $f=f(\boldsymbol{x}, t)$ is the single-particle distribution function in continuum, and $f^{(0)}$ the local Maxwellian. In Grad 13 moment theory, the set of moment equations are obtained by expanding the distribution function in Hermite polynomials (Grad, 1949a) in the reference frame moving with the local velocity. The choice of the Hermite polynomial as the expansion basis is that the expansion coefficients coincide with the hydrodynamic moments. Borrowing the idea here, we derive the LB equations by expanding the distribution function in Hermite orthogonal polynomials as:

$$
f=\omega(\boldsymbol{\xi}) \sum_{n=0}^{\infty} \frac{1}{n !} \boldsymbol{a}^{(n)}(\boldsymbol{x}, t) \mathcal{H}^{(n)}(\boldsymbol{\xi}),
$$

where $\mathcal{H}^{(n)}$ is the $n$-th Hermite polynomial, $\boldsymbol{a}^{(n)}$ the corresponding expansion coefficient, both rank- $n$ tensors.

First, recall that the hydrodynamic equations are obtained by taking the conserved moments of Eq. (6) (Huang, 1987). In terms of the hydrodynamic variables, they are:

$$
\begin{aligned}
& \frac{d \rho}{d t}+\rho \nabla \cdot \boldsymbol{u}=0 \\
& \rho \frac{d \boldsymbol{u}}{d t}+\nabla \cdot \boldsymbol{P}=0 \\
& \rho \frac{d \epsilon}{d t}+\boldsymbol{P}: \nabla \boldsymbol{u}+\frac{1}{2} \nabla \cdot \boldsymbol{S}=0,
\end{aligned}
$$

where the density $\rho$, fluid velocity $\boldsymbol{u}$, internal energy density per mass $\epsilon$, pressure tensor $\boldsymbol{P}$ and heat flux $\boldsymbol{S}$ are all velocity moments of $f$ :

$$
\begin{aligned}
\rho & =\int f d \boldsymbol{\xi} \\
\rho \boldsymbol{u} & =\int f \boldsymbol{\xi} d \boldsymbol{\xi} \\
\rho \epsilon & =\frac{1}{2} \int f|\boldsymbol{\xi}-\boldsymbol{u}|^{2} d \boldsymbol{\xi} \\
\boldsymbol{P} & =\int f(\boldsymbol{\xi}-\boldsymbol{u})(\boldsymbol{\xi}-\boldsymbol{u}) d \boldsymbol{\xi} \\
\boldsymbol{S} & =\int f|\boldsymbol{\xi}-\boldsymbol{u}|^{2}(\boldsymbol{\xi}-\boldsymbol{u}) d \boldsymbol{\xi} .
\end{aligned}
$$

The equations above are closed with the Chapman-Enskog asymptotic approximation procedure. At the zero-th order, the distribution derivation is approximated by the local Maxwellian, which yields at the macroscopic level the Euler equation. On substituting $f=$ $f^{(0)}+f^{(1)}$ into Eq. (6), we obtain the first correction as:

$$
f^{(1)}=-\tau\left(\frac{\partial}{\partial t}+\boldsymbol{\xi} \cdot \nabla\right) f^{(0)}
$$

On substituting the Hermite expansions of both $f^{(0)}$ and $f^{(1)}$ into the above equation, we have:

$$
\boldsymbol{a}_{1}^{(n)}=-\tau\left[\frac{\partial \boldsymbol{a}_{0}^{(n)}}{\partial t}+\nabla \boldsymbol{a}_{0}^{(n-1)}+\nabla \cdot \boldsymbol{a}_{0}^{(n+1)}\right],
$$


where $\boldsymbol{a}_{i}^{(n)}$ are the $n$-th Hermite expansion coefficients of $f^{(i)}$. To be noted that that the $n$-th expansion coefficient of $f^{(1)}$ only depend on the $n+1$-th coefficient of $f^{(0)}$. Since $f^{(1)}$ gives the correction at the NS level, we thus arrive at our first important conclusion: for Eq. (6) to have the NS level hydrodynamics, it is necessary and sufficient to retain all the low-order Hermite components in $f^{(0)}$ up to the order that is one higher that the highest moments of interest in $f^{(1)}$. To generalize, for Eq. (6) to recover the correct behavior a $n$-th moment at the $k$-th hydrodynamic approximation, it is necessary and sufficient to retain in $f^{(0)}$ all moments up to the $n+k$-th order.

Secondly, notice that for a finite Hermite series:

$$
f^{N}=\omega(\boldsymbol{\xi}) \sum_{n=0}^{N} \frac{1}{n !} \boldsymbol{a}^{(n)}(\boldsymbol{x}, t) \mathcal{H}^{(n)}(\boldsymbol{\xi}),
$$

any moment of $f^{N}$ can be written as:

$$
\int \boldsymbol{\xi}^{M} f^{N} d \boldsymbol{\xi}=\int \omega(\boldsymbol{\xi}) \frac{\boldsymbol{\xi}^{M} f^{N}}{\omega(\boldsymbol{\xi})} d \boldsymbol{\xi} .
$$

Now note that $\boldsymbol{\xi}^{M} f^{N} / \omega(\boldsymbol{\xi})$ is a polynomial in $\boldsymbol{\xi}$ of an order not exceeding $M+N$. Also note that for any polynomial in $\boldsymbol{\xi}, p^{n}(\boldsymbol{\xi})$, of a finite order $n$, there exist quadratures such that:

$$
\int \omega(\boldsymbol{\xi}) p^{n}(\boldsymbol{\xi}) d \boldsymbol{\xi}=\sum_{i=1}^{d} w_{i} p^{n}\left(\boldsymbol{\xi}_{i}\right),
$$

where $w_{i}$ and $\boldsymbol{\xi}_{i}, i \in\{1, \cdots, d\}$, are the weights and abscissas of the quadrature. We arrive at our second important conclusion: for any distribution that is a finite Hermite series of order $N$, its leading $M$ moments can be uniquely determined by its values on a set of discrete velocities provided the discrete velocities form a quadrature of a degree of precision of

$$
Q \geq M+N \text {. }
$$

Defining $f_{i}=w_{i} f\left(\boldsymbol{\xi}_{i}\right) / \omega\left(\boldsymbol{\xi}_{i}\right)$, the dynamics of the discrete distribution function values are governed by direct evaluation of Eq. (6):

$$
\frac{\partial f_{i}}{\partial t}+\boldsymbol{\xi}_{i} \cdot \nabla f_{i}=-\frac{1}{\tau}\left[f_{i}-f_{i}^{(0)}\right] .
$$

With these two conclusions, the LB equation can be considered as equivalent to the projection of the Boltzmann-BGK equation in the Hilbert space spanned by the leading Hermite polynomials. The truncation level, $N$, and the degree of precision of the discrete velocities as a quadrature, determines the highest order of the moments whose dynamics are contained in Eq. (22).

The remaining issues can be solved in this new theoretical framework. First, analysis of the accuracy of the truncation level reveals that all previous models slightly fall short in terms of the truncation order in recovering the full NS equation. To fully recover the momentum equation at the NS level, third order terms must be retained in $f^{(0)}$, which in turn requires a quadrature of at least sixth order. However most of the commonly used LB models, including the ones designed to eliminate the non-Galilean invariance by Qian and Zhou (1998), is only fifth order accurate. The consequence of this lack of accuracy is a small error term which causes the viscosity to be velocity-dependent. A similar observation can be made on the heat transport. Nie et al. (2008) showed that once sufficiently accurate equilibrium distribution and lattice are used, the LB is completely Galilean invariant. Secondly, the new derivation does not presume small Mach number. Instead, the orthogonal expansion in Hermite polynomials is valid for any finite Mach number, although the convergence speed deteriorates as Mach number is increased. Third, to include the effect of higher moments into the LB system, which is necessary in simulation of flows in finite Knudsen number regime, all we need to do is to increase the truncation level of the equilibrium distribution and expand the lattice so that the corresponding quadrature is sufficiently accurate.

Although the current LB formulation has many in common with Grad 13 moment theory, there are a number of important differences. First, in Grad 13 moment system, the distribution function is expanded in Hermite 
polynomials in a reference frame moving with the local velocity. Therefore, the moment equation has the same non-linear advection term as in all hydrodynamic equations, and the expansion convergence is independent on the local velocity or Mach number. In the LB system however, the expansion is in the laboratory reference so that the advection term is linear, but the convergence of the expansion is poor at high Mach numbers. Second, in Grad 13 moment system, the expansion coefficients are the state variables, and the governing partial differential equations are complex and differ substantially among all the moments. The enormous complexity has limited the use of the moment equation to highly idealized situations.

In summary, uniquely among all discretevelocity kinetic models, the LB equations can be shown analytically to converge asymptotically to the continuum Boltzmann-BGK equation as higher order moments are retained and more velocities used. Each level of truncation/quadrature accuracy corresponds to a level in hydrodynamic approximation. To include higher order hydrodynamic effects, it is only necessary to increase the level of truncation and the accuracy of the velocity set as a quadrature, but not the structural complexity. The equations are simple, explicit, easy to parallelize, and admit low-level modeling of complex fluid physics. It is for these reasons that we believe that LB offers a very promising simulation methodology for micro and nano flows.

\section{Micro and nano flows simulation}

Due to its kinetic nature, LB has naturally been considered for applications in simulation of micro and nano flows. As it is wildly accepted that in the slip regime, $10^{-3}<K n<10^{-1}$, the continuum hydrodynamic models, e.g., the NS equations, are still valid when equipped with proper slip boundary conditions, in earlier works, attentions have primarily focused on the study of the slip boundary conditions and the definition and scaling of the Knudsen number with respect to the relaxation time in the context of LB models (Nie et al., 2002; Lim et al., 2002; Niu et al., 2004; Lee and Lin, 2005; Ansumali et al., 2006). Sbragaglia and Succi (2005) and Ansumali et al. (2007) also obtained analytical LB solutions for simple channel flows. In most of these studies, the standard (NS level) LB models are used in the interior of the fluid and either the basic bounceback or the slightly more complex diffuse boundary conditions are used at the solid boundaries. Despite the apparent simplicity of this approach, and therefore some rather philosophical debate around its validity, it was generally concluded that at least in the slip regime, the slip at solid walls can be captured by LB to an extent ranging from good to excellent, while the bulk of the fluid still obeys the NS dynamics, e.g., having a parabolic velocity profile in channel flow. With the Knudsen number defined with the relaxation time as:

$$
K n(\tau-0.5) / H
$$

and the diffusive boundary condition used, the better results were achieved.

In another class of studies, the effect on wall-slip by the non-ideal fluid-wall interaction is modeled using multiphase LB equations. As discussed previously, the kinetic nature of the LB method allows the microscopic interaction to be modeled at the kinetic level. Taking advantage that, Harting et al. (2006) was able to reproduce in LB simulation the dependence of slip on the hydrophobicity of the channel walls and found constant slip for varying flow velocities.

More recently, numerical evidences start to appear on the convergence of LB to continuum Boltzmann-BGK in simulations of micro flows. Zhang et al. (2006) successfully demonstrated that the Knudsen paradox can be captured in LB simulation of channel flow. In this study the two-dimensional gravity-driven channel flow is simulated using both the D2Q9 model and a 21-speed high-order model which is capable of capturing fifth moment of a second order distribution. In addition, a "regularize" procedure is employed to limit the moments contained in the distribution function (not the equilibrium) to the orders that the lattice support. This is 
necessary to ensure the simulation results are isotropic. First, the isotropy of both models is tested in simulation of a decaying shear wave. As shown in Fig. 2, the decay rate in unregulated D2Q9 simulation depend on the orientation of the wave vector with respect to the lattice structure whereas the 21-speed model gives isotropic result. Second, it was found that without applying the regularize procedure, Knudsen's minimum exists in simulations using either D2Q9 or the 21-speed model. This is in consistent with the work of Ansumali. However, when the high- order components of the distribution function are filtered out, the solution using D2Q9 reverts back to the standard NS solutions whereas the Knudsen minimum persists in the solution of the 21speed model. From this numerical experiment, it can be concluded that the Knudsen minimum observed in LB simulation is caused by the high-order components independent of the implementation of the boundary conditions. The un-regularized distribution contains moments of all orders which explains why Knudsen minimum appears in simulation using basic LB models. However, the standard lattices are not accurate enough as a quadrature to support the dynamics of the high-order components to the extent that the macroscopic flow is isotropic whereas the high-order LB model can indeed capture the Knudsen effect accurately.

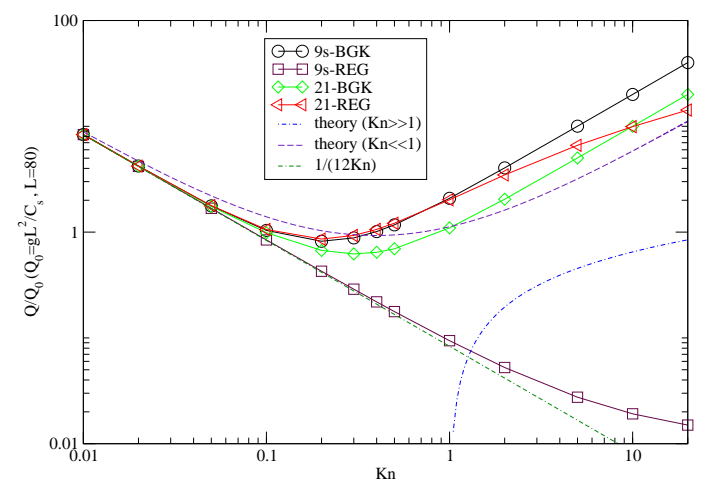

Fig. 2: Normalized mass flux as a function of Knudsen number in channel flow. Simulations with the standard D2Q9 and a high-order 21-speed model are carried out. To be seen is that while the standard D2Q9 model predicts the Knudsen minimum in the un-regularized version, but revert to the NS solution when the (un-isotropic) high-order components of the distribution function are removed in the regularized version. The high-order 21-speed model predicts the Knudsen minimum in both the regularized and un-regularized versions.

The accuracy of the high-order LB models is recently investigated by Kim et al. (2008). The accuracy of a number of two-dimensional high order lattices, mostly product quadratures derived from 1D quadratures up to 11th order, are evaluated in simulation of Couette and Poiseuille flows at finite Knudsen number. Simulation results on the velocity slip, the prediction of the Knudsen layer, and the mass flux are compared with the prediction of linearized Boltzmann equation and DSMC results. It was concluded that all high order LB models improve on the accuracy in finite Kn flows over the standard LB. With consistent definition of $\mathrm{Kn}$, the high order models give quantitatively accurate results up to $K n=$ $O(1)$ while the standard LB is only accurate up to $\mathrm{Kn}=0.05$. However, simulation results of all the high order LB models deviate from the prediction of the linearized Boltzmann and DSMC.

\section{Conclusions and Discussion}

In this presentation, the recent development of LB method as a computational tool for micro and nano flow simulation is reviewed. Theoretically, the LB equation hierarchy is shown to converge asymptotically to the continuum Boltzmann-BGK equation as the truncation level of the equilibrium distribution increased and more accurate lattices are used. Each level of this asymptotic approximation corresponds to a truncation in the Grad moment systems. As the Boltzmann equation is valid for flows at any Knudsen number, the LB hierarchy is argued to offer an efficient way for micro and nano flow computations. 


\section{References}

Abe, T., 1997. Derivation of the lattice Boltzmann method by means of the discrete ordinate method for the Boltzmann equation. J. Comp. Phys. 131, 241.

Alexander, F. J., Chen, S., Sterling, J. D., April 1993. Lattice Boltzmann thermohydrodynamics. Phys. Rev. E 47 (4), R2249-52.

Ansumali, S., Karlin, I., Arcidiacono, S., Abbas, A., Prasianakis, N. I., 2007. Hydrodynamics beyond Navier-Stokes: Exact solution to the lattice Boltzmann hierarchy. Phys. Rev. Lett. 98, 124502.

Ansumali, S., Karlin, I., Frouzakis, C. E., Boulouchos, K., 2006. Entropic lattice Boltzmann method for microflows. Physica A 359, 289-305.

Bhatnagar, P. L., Gross, E. P., Krook, M., May 1954. A model for collision processes in gases. I. Small amplitude processes in charged and neutral one-component system. Phys. Rev. 94 (3), 511-25.

Chen, H., Chen, S., Matthaeus, W. H., April 1992. Recovery of Navier-stokes equations using a lattice- gas Boltzmann method. Phys. Rev. A 45 (8), R5339-42.

Chen, S., Doolen, G., 1998. Lattice Boltzmann method for fluid flows. Ann. Rev. Fluid Mech. 30, 329-364.

Chen, Y., Ohashi, H., Akiyama, M., 1994. Thermal lattice Bhatnagar-Gross-Krook model without nonlinear deviations in macrodynamic equations. Phys. Rev. E 50, 27762783.

Doolen, G. D. (Ed.), 1989. Lattice Gas Methods for Partial Differential Equations. Addison Wesley, Redwood City, California.

Frisch, U., Hasslacher, B., Pomeau, Y., 1986. Lattice-gas automata for the Navier-Stokes equation. Phys. Rev. Lett. 56, 1505.
Grad, H., 1949a. Note on N-dimensional Hermite polynomials. Commun. Pure Appl. Math. 2, 325.

Grad, H., 1949b. On the kinetic theory of rarefied gases. Commun. Pure Appl. Math. 2, 331.

Harting, J., Kunert, C., Herrmann, H. J., 2006. Lattice Boltzmann simulations of apparent slip in hydrophobic microchannels. Europhys. Lett. 75 (2), 328-334.

Házi, G., Kávrán, P., 2006. On the cubic velocity deviations in lattice Boltzmann methods. J. Phys. A 39, 3127-36.

He, X., Luo, L., 1997. A priori derivation of the lattice Boltzmann equation. Phys. Rev. E 55 (6), R6333.

Higuera, F. J., Jimenez, J., Aug. 1989. Boltzmann approach to lattice gas simulations. Europhys. Lett. 9 (7), 663-8.

Huang, K., 1987. Statistical Mechanics, 2nd Edition. John Wiley \& Sons, New York.

Kim, S. H., Pitsch, H., Boyd, I. D., 2008. Accuracy of higher-order lattice Boltzmann methods for microscale flows with finite knudsen numbers. J. Comp. Phys. 227, 865571.

Lee, T., Lin, C.-L., 2005. Rarefaction and compressibility effects of the lattice-Boltzmannequation method in a gas microchannel. Phys. Rev. E 71, 046706.

Li, W., Wei, X., Kaufman, A., 2003. Implementing lattice Boltzmann computation on graphics hardware. The Visual Computer 19, 444-56.

Lim, C. Y., Shu, C., Niu, X. D., Chew, Y. T., 2002. Applications of lattice Boltzmann method to simulate microchannel flows. Phys. Fluids 14 (7), 2299.

McNamara, G., Alder, B., March 1993. Analysis of the lattice Boltzmann treatment of hydrodynamics. Physica A 194 (1-4), 218 28. 
McNamara, G. R., Garcia, A. L., Alder, B. J., Oct 1995. Stabilization of thermal lattice Boltzmann models. J. Stat. Phys. 81 (1-2), 395-408.

McNamara, G. R., Garcia, A. L., Alder, B. J., 1997. A hydrodynamically correct thermal lattice Boltzmann model. J. Stat. Phys. 87 (5/6), 1111-21.

McNamara, G. R., Zanetti, G., 1988. Use of the Boltzmann equation to simulate latticegas automata. Phys. Rev. Lett. 61, 2332.

Nie, X. B., Doolen, G. D., Chen, S., April 2002. Lattice-Boltzmann simulations of fluid flows in MEMS. J. Stat. Phys. 107 (1/2), 279-89.

Nie, X. B., Shan, X., Chen, H., 2008. Galilean invariance of lattice boltzmann models. Europhys. Lett. 81, 34005.

Niu, X. D., Shu, C., Chew, Y. T., 2004. A lattice Boltzmann BGK model for simulation of micro flows. Europhys. Lett. 67 (4), 600606.

Orszag, S. A., Yakhot, V., April 1986. Reynolds number scaling of cellular-automaton hydrodynamics. Phys. Rev. Lett. 56 (16), 16911693.

Qian, Y.-H., d'Humieres, D., Lallemand, P., Feb. 1992. Lattice BGK models for NavierStokes equation. Europhys. Lett. 17 (6), 47984.

Qian, Y.-H., Orszag, S. A., Jan. 1993. Lattice BGK models for the Navier-stokes equation: nonlinear deviation in compressible regimes. Europhys. Lett. 21 (3), 255-9.

Qian, Y.-H., Zhou, Y., 1998. Complete Galilean-invariant lattice BGK models for the Navier-Stokes equation. Europhys. Lett. 42 (4), 359-364.

Sbragaglia, M., Succi, S., 2005. Analytical calculation of slip flow in lattice Boltzmann models with kinetic boundary conditions. Phys. Fluids 17, 093602.

Shan, X., He, X., January 1998. Discretization of the velocity space in solution of the Boltzmann equation. Phys. Rev. Lett. 80, 65.

Shan, X., Yuan, X.-F., Chen, H., March 2006. Kinetic theory representation of hydrodynamics: A way beyond the Navier-Stokes equation. J. Fluid Mech. 550, 413-441.

Struchtrup, H., 2002. Heat transfer in the transition regime: Solution of boundary value problems for Grads moment equations via kinetic schemes. Phys. Rev. E 65 (4), 041204.

Succi, S., August 2001. The Lattice Boltzmann Equation for Fluid Dynamics and Beyond. Numerical Mathematics and Scientific Computation. Oxford University Press.

Wolfram, S., 1986. Cellular automaton fluid 1: Basic theory. J. Stat. Phys. 45 (3/4), 471-526.

Zhang, R., Shan, X., Chen, H., 2006. Efficient kinetic method for fluid simulation beyond the Navier-Stokes equation. Phys. Rev. E 74, 046703. 